Print ISSN: $2735-4121$

Online ISSN : $2735-413 X$

\title{
EFFECT OF INSTRUCTIONAL LEARNING PACKAGE ON KNOWLEDGE AND SELF-CARE OF DIABETIC PATIENTS AT MANSOURA UNIVERSITY HOSPITAL \\ ${ }^{1}$ Ahmed Mohamed Esam Belal, ${ }^{2}$ Amany Mohamed Shebl Abd Ellateef, and ${ }^{3}$ Hanan Abu Bakr Mohamed
}

(1)Assisstant Lecturer, (2) professor, (3) Assistant professor

1,2,3, Medical-Surgical Nursing Department, Faculty of Nursing-Mansoura University

E-mail of corresponding author: ahmedbelal88@yahoo.com

\begin{abstract}
:
Chronic metabolic disorder diabetes mellitus is a fast-growing global problem with huge social, health, and economic consequences. It is estimated that in 2010 there were globally 285 million people (approximately 6.4\% of the adult population) suffering from this disease. Therefore, the aim of the study was to evaluate the effect of instructional learning package on knowledge and self-care of diabetic patients at mansoura university hospital. Setting The study was conducted at endocrine department at specialized medical hospital, Mansoura University, Egypt. Tools, three tools were used (1) Demographic characteristics and medical history interview sheet, (2) Patients' knowledge Questionnaire for DM, (3) Self-care of diabetic patient's questionnaire.

Methods Quasi-experimental research design was used in this study. Knowledge and Self-care of diabetic patients' questionnaire was designed after the literature review. A purposive sample of sixty adult male and female patients were included in this study admitted through a period of 6 months from January, 2019 to June, 2019 diagnosed with diabetes mellitus confirmed by diagnosis in patient file. Results The results revealed that the total mean score for patient knowledge about disease and patient self-care were higher in the post intervention more than the pre intervention. The total mean score for patient foot care was higher in the post intervention than the pre intervention. The total mean score for insulin self-administration was higher in the post intervention than the pre intervention.
\end{abstract}

Key words: Instructional learning Package, knowledge, self-care. Diabetes.

\section{Introduction:}

Diabetes happens when glucose in blood rises above normal range. Blood glucose is the fuel of body and comes from feeding. Insulin is hormone helps in converting excess blood glucose to energy. Excess blood sugar can cause many problems. ${ }^{(1)}$

There are two types of diabetes, type 1 which is auto immune disorder cause destruction of beta cells and called juvenile diabetes and another type 2 due to insulin resistance occurring in adults in obese patients and non-insulin dependent diabetes (2)
Improving patient knowledge is an important issue in minimizing complications of diabetes and improving life style of patients, so an educational program for diabetic patients can help in making patient self-care better and this can be achieved through television programs, booklet containing clear pictures and through workshops. (3)

Diabetes can affect on diabetic patients leading to many complications in heart ,kidney ,nerves and eye so selfcare is important in minimizing diabetes complications as smoking cessation 
,exercise, follow up and therapeutic diet. (4)

\section{Aim of study:}

Evaluate the effect of instructional learning package on knowledge and selfcare of diabetic patients at mansoura university hospital.

\section{Research hypothesis:}

Patients who attend educational sessions related to diabetes mellitus will be more knowledgeable about disease.

Self-care of diabetic Patients who attend educational sessions related to diabetes mellitus will improve.

\section{Subjects \& Methods}

\section{1-Study Design:-}

Quasi-experimental research design was used in this study

\section{2- Setting:-}

This Study was conducted in endocrine department at specializedmedical hospital at Mansoura University.

\section{3- Subjects}

A purposive sample of sixty adult male and female patients admitted through a period of six months diagnosed with diabetes mellitus.

\subsection{1: Inclusion criteria:}

- Diabetic patients Type I and type II aged from 20-60 years old.

- Both sexes.

- Willing to participate voluntarily in the study and give consent

- Able to communicate and verbalize their needs.

- Patients who have no complications of diabetes

- Patients with controlled diabetes

\subsection{2: Exclusion criteria: -}

- Unconscious patients.

- Patients with psychosis and mental disabilities.
- Patients who refuse to participate 4.4-Tools: and give consent in the study.

Tool I: Demographic Characteristics and medical history interview sheet

This tool was developed by the researcher after reviewing the related recent literature and consists of two main parts

Part A:

This part includes eight demographic characteristics of patients such as (age, sex, marital status, level of education, social status, living place, occupation, and income) used one time at the beginning of study to gain basic data about patients.

Part B: Medical history of the patient, this part includes three items such as duration of disease, type of diabetes, current therapeutic regimen including medication, diet, exercise and follow up. Tool I1: patient's knowledge Questionnaire for DM.

This tool was adapted and modified by the researcher based on reviewing recent literature. It includes (43 items); patient knowledge about disease (6 items), patient knowledge about causes (9 items) , patient knowledge about symptoms (14 items) ,patient knowledge about complications (8items) ,patient knowledge about therapeutic regimen (6items) using pre and posttest.

\section{Scoring system}

Answering questions requiring "Yes," "No," A correct answer was scored $=1$; while an incorrect, unknown answer was scored $=0$. The scores obtained for each question were summed up to get the total score for patient's knowledge about diabetes mellitus. Higher scores indicated more knowledge about diabetes mellitus and vice versa. The level of knowledge was classified 
according to high, moderate, and mild for overall knowledge and in each knowledge section. A score of $75 \%$ was classified as "High knowledge", from $74 \%$ to $50 \%$ was classified as "Moderate knowledge", and a score $<50 \%$ was classified as "Mild knowledge". Tasha. (2006).

Tool II1: Self-care of diabetic patient's questionnaire:

This tool was adopted and modified by the researcher based on reviewing recent literature to assess patient' self-care including (dietmedication-exercise-follow-up-self

monitoring -foot care- Insulin selfinjection) using pre and posttest.

\section{Scoring system}

Answering questions requiring "Yes," "No," A correct answer was scored $=1$; while an incorrect answer was scored $=0$. . The scores obtained for each question were summed up to get the total score for patient's self-care about diabetes mellitus. Higher scores indicated more self-care and viceversa. The level of self-care was classified according to high, moderate, and mild for overall self-care and in each self-care section. A score of $75 \%$ was classified as "High self-care", from $74 \%$ to $50 \%$ was classified as "moderate self-care", and a score $<50 \%$ was classified as "mild selfcare".Akhter.(2010).

\section{5- Methods:}

1. Permission should be gotten to start study obtained by the researcher from responsible authorities at endocrine department of Specialized Medical Hospital, Mansoura University.

2. Verbal explanation of the nature and aim of the study was performed to medical \&nursing staff.

3. Tools (I), (II), (III) was developed and adopted by the researcher after thorough reviews of related literature.

4. Tools were tested for its content validity by a jury of experts in the field of medicine and nursing faculty staff, and any required modifications will be done.

5. Tools were tested for its reliability using test - retest measurement and Cronbach's alpha.

6. A pilot study was carried on 7 patients $(10 \%)$, before starting the data collection to test the clarity, feasibility, applicability and time of the tools and to make the necessary modification; these patients were not be included in the study.

7. Human rights and ethical consideration, prior to the pilot of this study, an approval was obtained from each participating patient prior to his /her inclusion into the study .clarification of the nature and purpose of the study was done on the interview with each client. The researcher emphasized participation is absolutely voluntary and confidential. Anonymity, privacy, safety and confidentiality will be absolutely assured throughout the whole study and the right to withdraw from the study at any time.

8. Each patient was interviewed individually in order to collect the necessary data using the study tools.

\section{Data collection}

Data was collected by the researcher during the period from January, 2019 to June, 2019.This study was carried out over three phases: assessment phase, implementation phase, and evaluation phase.

The first phase: assessment phase Assessment phase included assessment of demographic data of patients, diabetic patients knowledge and 
self-care using tool (I), tool (II) \&tool (III) that carried out through two months to determine patient knowledge and selfcare gap, every patient was interviewed individually by the researcher to assess patient knowledge about disease ,causes ,signs \&symptoms ,complications and therapeutic regimen and self-care level including medication ,diet, exercise, smoking cessation, follow up, foot care and insulin self-administration through answering pretest questionnaire questions by patients and filled by researcher to determine patient defects and gaps in knowledge and self-care and try to improve it through an educational programme given to patients and developing an educational booklet that can meet patient needs in knowledge and self-care .

The second Phase (Implementation phase):

Implementation phase included implementing educational program according to patient's gap of knowledge and self-care and after reviewing recent literature to provide patients with information about diabetes mellitus, in order to improve their knowledge and self-care level to prevent the complications of the disease through giving an educational booklet for each patient. Educational programme included two parts theoretical and practical part.

Theoretical part: Theoretical part included educating patients about diabetes mellitus, causes, symptoms, complications, importance of regular glucose monitoring, dose of medication, proper diet, and importance of exercise \&follow up, foot care \& insulin injection.

Practical part: Practical part included educating patients about selfcare behaviors such as foot care, and self-insulin administration.

\section{$\underline{\text { Number of sessions }}$}

The educational programme designed for this study has been implemented through three sessions for each patient, one session per day, each session lasted from 30-40 minutes including from seven to eight patients. According to content provided, three sessions were provided weekly and be conducted either individually for patients or also for group according to the availability of time and place divided as

-One session was given to cover knowledge about diabetes mellitus, causes, signs \&symptoms, complications, importance of glucose monitoring.

- One session was given to cover patient knowledge about self-care including diet, exercise, follow up, medication, foot care, insulin injection

-One session was given to teach patient foot care and self-insulin injection

\section{The third Phase (Evaluation phase):}

Evaluation phase was carried out after two months from implementation phase which included post assessment of diabetic patients' knowledge and selfcare using Tool (II) \&Tool III to evaluate the effect of this programme on patient knowledge and self-care. The patient answered the same questions present in the first phase (assessment phase).

\section{5- Results:}

The data collected were analyzed statistically and the results were categorized into five main parts which are: demographic characteristics, Patient knowledge about disease pre and post intervention, patient self-care about disease pre and post intervention, patient foot care, patient insulin administration pre and post intervention 
Table (5.1): Demographic characteristics of the studied patients:

Males were more prevalent in the study sample than females, they constitute (70 $\%)$ of the patients, while only (30\%) were females ,the age of the studied patients ranged from 20 to less than 60 years. More than half of patients $(65 \%)$ were aged from $>50$ years old, $(20 \%)$ from 40:50years old, Regarding the educational level, half of patients $(50 \%)$ were technical school and only $(25 \%)$ were universal education.

\begin{tabular}{|c|c|c|c|}
\hline Demographic data & & $\begin{array}{c}N \\
(60)\end{array}$ & $\%$ \\
\hline \multirow{2}{*}{ Sex } & Male & 42 & 70.0 \\
\hline & Female & 18 & 30.0 \\
\hline \multirow{4}{*}{ Age group } & $20: 30$ & 2 & 3.3 \\
\hline & $30: 40$ & 7 & $11 . \overline{7}$ \\
\hline & $40: 50$ & 12 & 20.0 \\
\hline & $>50$ & 39 & 65.0 \\
\hline \multirow{3}{*}{ Education level } & Illiterate & 14 & 23.3 \\
\hline & Technical school & 30 & 50.0 \\
\hline & University college & 16 & 25.0 \\
\hline \multirow{2}{*}{ living place } & urban & 17 & 28.3 \\
\hline & rural & 43 & 71.7 \\
\hline
\end{tabular}

Table (5.2): Total mean scores for patient knowledge in the pre and post intervention

\begin{tabular}{|c|c|c|c|c|}
\hline \multirow{2}{*}{ Total Mean \pm Sd } & \multicolumn{2}{|c|}{ Pre intervention } & \multicolumn{2}{c|}{ Post intervention } \\
\cline { 2 - 5 } & Mean \pm Sd & Median & Mean \pm Sd & Median \\
\cline { 2 - 5 } & $25.7 \pm 11.1$ & $25(9-43)$ & $38.8 \pm 8.6$ & $42.5(6-43)$ \\
\hline
\end{tabular}

The total knowledge mean score of patient knowledge about disease improved from (25.7 $\pm \mathbf{1 1 . 1})$ in the pre intervention to $(\mathbf{3 8 . 8} \pm \mathbf{8 . 6})$ in the post intervention.

\section{Table (5.3): Total mean scores for patient self-care in the pre and post intervention}

\begin{tabular}{|c|c|c|c|c|}
\hline \multirow{2}{*}{$\begin{array}{c}\text { Total mean self- } \\
\text { care score of } \\
\text { patients in the pre } \\
\begin{array}{c}\text { and post } \\
\text { intervention }\end{array}\end{array}$} & \multicolumn{2}{|c|}{ Pre intervention } & \multicolumn{2}{c|}{ Post intervention } \\
\cline { 2 - 5 } & $8.8 \pm 2.7$ & $8.5(4-14)$ & $10.1 \pm 2.0$ & $10(5-14)$ \\
\hline
\end{tabular}

The total self-care mean score of the patients in the pre intervention phase is $\mathbf{8 . 8} \pm \mathbf{2 . 7}$, which increased to $\mathbf{1 0 . 1} \pm \mathbf{2 . 0}$ in the post intervention. 
Table $(5,4)$ : Total mean score for patient foot care pre and post intervention

\begin{tabular}{|c|c|c|c|c|}
\hline \multirow{3}{*}{$\begin{array}{l}\text { Total mean score } \\
\text { for patient foot } \\
\text { care pre and post } \\
\text { intervention phase }\end{array}$} & \multicolumn{2}{|c|}{ Pre intervention } & \multicolumn{2}{|c|}{ Post intervention } \\
\hline & $\operatorname{Mean} \pm$ Sd & Median & $\operatorname{Mean} \pm$ Sd & Median \\
\hline & $5.9 \pm 4.2$ & $6(1-16)$ & $11.2 \pm 4.4$ & $12(2-19)$ \\
\hline
\end{tabular}

It was observed that the total mean score for patient foot care in the pre intervention stage is 5.9 \pm 4.2, which increased in the post intervention stage to $11.2 \pm 4.4$.

Table $(5,5)$ : Total mean score for patient insulin self-administration pre and post intervention

\begin{tabular}{|c|c|c|c|c|}
\hline \multirow{3}{*}{$\begin{array}{l}\text { The total mean score } \\
\text { for insulin self- } \\
\text { administration of } \\
\text { patients pre and post } \\
\text { intervention }\end{array}$} & \multicolumn{2}{|c|}{ Pre intervention } & \multicolumn{2}{|c|}{ Post intervention } \\
\hline & Mean \pm Sd & Median & $\operatorname{Mean} \pm$ Sd & Median \\
\hline & $2.5 \pm 3.6$ & $0(0-9)$ & $7.9 \pm 2.5$ & $9(0-9)$ \\
\hline
\end{tabular}

It was observed that the total mean score for insulin self-administration of patients in the pre intervention stage is $\mathbf{2 . 5} \pm \mathbf{3 . 6}$, which increased in the post intervention stage to $\mathbf{7 . 9} \pm \mathbf{2 . 5}$

\section{6- Discussion}

When examining the results of knowledge scores of the studied sample, the current study revealed that the total mean knowledge score of study sample pre implementation of instructional learning package was unsatisfactory and this may be due to patient level of education, living place and absence of patient advisor, while in the post implementation of instructional learning package, there is an improvement in total mean score of patient knowledge and this is may be due to presenting educational booklet for each patient with

Clear information and simple language, in addition to interest of patients to know more information about the disease.

These findings are in agreement with (TeganyJ, 2010) who reported significant improvement in diabetic patient knowledge after implementation of instructional learning package.

Also these findings are in agreement with (Youssef, 2014) who showed significant improvement of diabetic patient knowledge level after Implementation of instructional learning package.

Furthermore (Woodard c.m, 2014) which reported that the total mean knowledge of diabetic patients improved in the post intervention compared to pre intervention.

In addition to (Norris SL \& Engelgau MM, 2014) found that total mean of patient knowledge about diabetes improved in the post intervention compared to pre intervention.

On the other hand, this result does not agree with (Eldriny, 2012) which reported lack of knowledge related to 
disease found in subjects with other chronic illnesses in Egypt after implementation of instructional programme.

Also, this result is not congruent with (Fleury F, 2018) that demonstrated that most of patients had unsatisfactory level of knowledge after implementation of instructional learning package, this difference between the two study results may be due to difference in patient level of education, patient setting, culture and patient interest and willingness to know about the disease to improve their knowledge.

Regarding patient self-care, the results of the present study showed that the total mean self-care score of patients improved after implementation of instructional learning package more than being in pre intervention and that is may be due to lack of standardized patient education about self-care and difference in level of education between patients in the pre intervention, but after implementation of instructional learning package there was an improvement of patient self-care and this may be due to continuous demonstration ,redemonstration during sessions, practical content and instructional learning booklet with pictures .

It was found that our findings agree with findings of (Abd el Salam, 2010) who stated that there is an improvement in patient self-care after implementation of educational learning package compared to pre intervention .

Also it was found that (Refaii , 2011) stated an improvement in patient self-care score in the post implementation compared to pre implementation and this finding agrees with our results in this study.

This finding is in agreement with significant improvement in patient selfcare after implementing instructional learning programme

In addition to (Taylor, 2010) who mentioned that there is a significant improvement in patient self-care in the post implementation compared with pre implementation that agrees with our findings

Furthermore, (Ramos ,2007) stated significant improvement in patient wound care in the post implementation compared with pre implementation that matches with our results in this study

On the other hand, it was stated that the majority of patients were compliant with therapeutic regimen in the pre intervention according to (Gaddi $\mathbf{A V}, \mathbf{2 0 1 9})$ that disagrees with our results and this difference may be due to difference in level of patient education and culture between the two studies.

\section{7-Conclusion:}

- The total mean score for patient knowledge about disease was higher in the post intervention more than the pre intervention.

- High educated patients had the highest knowledge level, followed by technical school and the lowest knowledge level for illiterate patients in the pre intervention.

- The total mean score for patient selfcare was higher in the post intervention more than the pre intervention.

- The total mean score for patient foot care was higher in the post intervention than the pre intervention.

- The total mean score for insulin selfadministration was higher in the post intervention more than its being in the pre intervention. 


\section{8- Recommendations}

In the light of the findings of the current study, the following recommendations are suggested:

\section{For health care providers:}

- An educational program should be put in priority at each healthcare center and hospital

- Increase cooperative efforts between faculty of nursing staff and hospital managers to offer continuous health education about chronic diseases for all health care providers.

- Instructions should be given by health care providers about measures to help patients be compliant with prescribed therapeutic regimen.

\section{For patients:}

- Development of educational program for patients with diabetes mellitus disease to improve patient's knowledge about disease and therapeutic regimen and to be familiar with different aspects of treatment.

- Continuous health teaching using T.V programs, video tapes, and brochures to enhance patient's adherence with therapeutic regimen.

- Organizing patient's centered workshops to teach about diabetes mellitus disease and to enhance patient's knowledge and adherence to therapeutic regimen.

- Encouragement of socialization and friendship as this can enhance patient's adherence.

- Patients should be instructed about lifestyle modification as proper diet, performing exercise,

\section{9- References}

1-Abdel Salam. (2010): Designed Nursing Clinical Pathway Guidelines on cardiac pacing patient outcome, faculty of nursing, Cairo University, 32(2), PP 21-50.

2-Eldriny. (2012): Patients with myocardial function, factors effect on adherence to therapeutic regimen Master thesis in medical surgical department .faculty of nursing, Ain Shams University .36(1):pp 35-79 .

3-Fleury F. (2018): Improved glycaemic control by addition of glimepiride to metformin monotherapy in type 2 diabetic patients. Diabet Med. 18(10):828-34.

4-Gaddi A.(2019): Anti thrombotic effects of rosiglitazone-metformin versus glimepiride-metformin combination therapy in patients with type 2 diabetes mellitus and metabolic syndrome. Pharmaco therapy. ;25 (5):637-45.

5- Norris SL \& Engelgau MM. (2014): Effectiveness of self management training in type 2 diabetes; can compliance with pharmacologic treatment for diabetes disease be managed? Am J Prev Med 18:253-261.

6-Starr B. (2017): Improving Outcomes for Patients with Chronic Disease: The Medication Adherence Project (MAP). Cardiovascular Disease Prevention and Control Program.

7- Taylor,c.(2010) : A literature review Comparing The experience and needs of adult patients with permanent pacemakers and implantable cardiovascular Defibrillation .Journal of clinical Nursing, (19) 15:16 .

8-Tegany, J.(2010) : Introduction about needs and knowledge of diabetic patients .Journal , ,(19) 15:16 ,pp. 851-858.

9-Yousef,G.(2014) : Effect of educational program on quality of life of patients with permanent 
pacemaker, faculty of nursing, Ain Shams university ,22(9),PP.44-46.

10- Ramos.(2007): Nursing care of the cardiac surgical patient,7(ed) Bpston :kluwer Academic publisher,p.897.

11- Refaii A.S.(2011) : Impact of A Designed clinical pathway Guidelines on acute muocardial infraction patient outcome at Benha university hospital s.Faculty of Nursing ,33(12) .

12-Richard LG(2010) : US nurses perceptions of their role in diabetes care: results of the cross - national Diabetes Attitudes Wishes and Needs (DAWN) study . Diabetes Educ ; 33 : $152-162$.

13-Rippe RS. (2010): Manual of intensive care medicine (5th
ed.).Wolters

Kluwer

Health/Lippincott Williams \&

Wilkins. p. 549. .

14- Samith Shetty A.(2015) : Nanditha A. Primary prevention of type 2 diabetes in South Asians-challenges and the way forward. Diabet Med. ;30:26-34.

15-Sarwar N.(2016) : a collaborative meta-analysis of 102 prospectivestudies. Lancet ;375:221522.

16- Singh NP.(2017) :Effect of addition of either sitagliptin or pioglitazone in patients with uncontrolled type 2 diabetes mellitus on metformin: A randomized controlled trial. J Pharmacol Pharmacother. 4(1):2732. 